\title{
Estimating Traffic Intensity at Toll Gates Using Queueing Networks
}

\author{
Vincent O. R \\ Department of Computer Science \\ Federal University of Agriculture \\ Abeokuta, Nigeria
}

\author{
Olayiwola O. E. \\ Department of Computer Science \\ Federal University of Agriculture \\ Abeokuta, Nigeria
}

\author{
Kosemani O. O. \\ Department of Computer Science \\ Federal University of Agriculture \\ Abeokuta, Nigeria
}

\begin{abstract}
Traffic information generation is a routine-like operation that is done on a daily basis at any public gate. A toll gate is a public roadway by which people enter and leave a public organisation. The existing models give premium consideration to security over prompt services and as such associated with processes that have high cost of implementation, inaccuracy from complex method as well pose other technical problems such as delay. This research presents an automated procedure for monitoring traffic at toll gates to give the best compromise among the conflicting objectives of payment, security and good services. The system gathers information about the traffic situation with respect to the license plate number captured from each vehicle that passes through the toll gate and as well captures data such as arrival speed, arrival time and date and uses this data as input to generate traffic report/information on a daily basis. Experimentally the system shows that it can effectively capture the vehicle video and detect the license plate in day time, showing accuracy of about $85 \%$ to $90 \%$, practical results based on actual data are included.
\end{abstract}

General Terms: Image processing; Artificial intelligence; Information Engineering

Keywords-Toll Gates; Queueing Networks; Traffic intensity; Delay and Image detection

\section{INTRODUCTION}

A toll gate is a point of entry to a space enclosed by walls, or a moderately sized opening in some sort of fence. Gates may prevent or control the entry or exit of individuals, or they may be merely decorative. The main advantage of a toll gate is the opportunity to keep track of vehicles plying the highways, bridges, and tunnels on which the system is installed. The system enhances the collection of entrance payments which is done either manually or electronically. The manual mode of payment is considered primitive because it poses problems such as congestion at toll gates, especially during festive seasons when traffic tends to be heavier than normal [1]. This incontinency results in fatigue and inaccuracy in the automation of the system.

On the other hand, the electronic entrance system executes automatic payments using wireless communication without long stoppings at the express way. The problems with electronic system are the reflected, distracted, shadowed waves experienced by canopy, wall, or, booth and interference waves from neighboring lanes which may constitute threats to the operations of the system [2].
With the increasing number of vehicles on roads, traffic at gates could no longer be estimated manually but rather electronically. Electronic toll gates also help in enforcing laws and traffic rules for smooth flow of it. The number of vehicles moving in and out at different speeds and the timing are basic data that could generate information about the traffic situation. Entrance-gates are located majorly on freeways and parking structures for general checking purposes most importantly for payments and security. Toll gates are enabled with traffic management systems to check for vehicles moving at higher speeds. The purposes of creating toll gates were achieved at the expense of vehicle owners. The important question which has over the years not been answered is what are the implications of toll gate on vehicle owners and the society at large?

The literatures on toll gates are in several categories; research works either extract the license plate of vehicles for tracking or for estimating vehicles speed. Many papers analysed low frame-rate video taken from an un-calibrated camera and estimate mean traffic speed for tracking of vehicles [3]. Others dwelt on plate detection. A research presented an algorithm that allows automatic ID measurements and subsequent estimation of vehicle speed from single un-calibrated images [4]. Some use canny edge detection to detect vehicles speed [5], others used morphological operators to detect vehicle number plate from the videos of the vehicles in different illumination environments, but the central idea of license plate extraction is through video [6]. Another developed traffic monitoring using vehicle-based sensors of taxi with two traffic status estimation algorithms adopted: link-based and the vehicle based on sparse and incomplete information [7]. However, the sensors were always set with long sampling interval because of communication cost saving and network congestion avoidance.

Xie proposed a Privacy Awareness Monitoring System (PAMS) which works as aggregate query processor to protect the location privacy of drivers as it anonymizes the IDs of vehicles [8]. Though PAMS answers the problems of high queries and accuracy and also achieves good balances among privacy, accuracy and efficiency for traffic monitoring, purpose-centred mission were observed which were always aimed to achieve security over other inconveniences.

This work presents a system for license plate extraction from a video frame. It focuses on extracting general information of a vehicle arriving at toll gate, information such 
as its arrival speed and license plate number of vehicle and these information combined to estimate the traffic intensity at the gate given a sequence of real-time traffic videos that is taken from a camera strategically installed in the toll gate environment to capture the video of the activities of the booth when the vehicles pass through and generated images from which license plate number are extracted and then processed. The work solves the problem of inaccuracy and fatigue resulting from manually generating traffic information at toll gates as well as high cost of installation and maintenance of existing automated systems. The motivation of the work is to find a cost effective method to detect the vehicles' arriving speed, and their identification number, and using all these data as information to estimate the traffic intensity at the booth. The paper is arranged as follows: Section 2 presents the review of literature while section 3 describes the traffic monitoring system with queueing theory. Section 4 gives the results and evaluation, Section 5 concludes the work.

\section{RELATED METHODS}

\section{A. Automated Toll Gate System}

Automated Toll gate System is considered the most sophisticated entrance roads in the world [9]. Cameras are equipped with Optical Character Recognition (OCR). The OCR cameras are used to capture license plate numbers of vehicles without transponders. The entrance bill is then sent directly to the registered address of the vehicle owners. There are two laser beam scanners in the system which is placed above the roadway to detect the types of vehicles passing through the toll gate. The toll gate system is considered to bear a very high infrastructure cost, and the users are the ones who help recover the cost through increments in their entrance bills.

ATG is said to use a combination of mobile telecommunication technology (GSM) with satellite-based Global Positioning System (GPS). Using GPS technology, the distance driven in kilometers can be estimated, and use to calculate the entrance fees and rates, and then transmit the information to the NATCS computer centre. Each vehicle is charged from the highway entrance up until the end of the highway. In order to identify the plate numbers of trucks, the system has control gates equipped with digital short range communication (DSRC) detection equipment and high resolution cameras [10,24]. The system is considered expensive due to the technical specifications which incur high cost for motorists.

Other systems include TouchNGo and SmartTAG[11]. This system uses IR technology, making it very vulnerable to failure. It also has high cost of implementation since users have to own two-piece tag required for this system. Passive RFID technology based toll gate system is another automated system that guarantees increased efficiency, since RFID is considered a highly stable technology with the elimination of human interaction in systems based on this technology [1].

\section{1) Smartcard Based Toll Gate Automated System}

It is observed that the use of contact type smart cards cannot be underestimated in the world of technology because it is being utilized for different purposes. The latest technology trends introduced contactless smartcards. They work on the $R F$ frequencies. With the help of these smartcards there is no need to insert the smartcard in the reader, the reader reads the smartcard from the distance, and both the smartcard and the smartcard reader will transmit and receive signals which led to mutual transfer of information to other devices. It is considered faster than the contact based smart cards [2]. The Smartcard based toll gate automated system is considered effective and efficient since the card is recharged with some amount and whenever a person wants to pay the toll gate tax, just needs to insert the smart card and deduct amount using keypad, the system is security conscious since there is no need to carry cash. But it is considered expensive to install and maintain [2].

\section{2) Rfid Based Toll Gate Automated System}

Radio Frequency Identification (RFID) is an automated identification technology which uses Radio Frequencies between $30 \mathrm{kHz}$ and $2.5 \mathrm{GHz}$ to identify objects remotely. The RFID Automatic toll gate system is designed to automatically detect the identities of the vehicles and perform the billing in accordance with the identity of each vehicle as pre-recorded in the database [12]. The system could automatically open and close and automatically emails the owners of the vehicles. These were the major achievements of the system. Other features are the ability to track vehicles and connect database remotely.

In spite of these, the system has failed in some of the required criteria because it did not yield the required result due to lack of resources and high cost of implementation. For instance, remote database connection needed a pre-set Virtual Private Network and automatic synchronizing software which will be readily available $[12,28]$.

\section{B. License Plate Detection}

The development of a reliable and accurate License Plate Recognition (LPR) system cannot be underestimated in view of its potential application in traffic monitoring systems and highway entrance collection. LPR systems have recently attracted considerable interest as part of an Intelligent Transport System. While much commercial work has been done for Iran, Korean, Chinese, European and US license plates, little work has been done in LPR systems for developing country such as Nigeria [13]

The central idea of the license plate extraction is to detect vehicle plate number from video Existing systems are based on four modules. In the first module, the camera captures video of the vehicle. In the second module, the video is converted into frames by using MATLAB operations. The third module converts frames into images. Finally, in the last module by using canny edge detection and morphological operator's vehicle number plate is extracted. The main advantage of this technique is that from $10 \mathrm{sec}$ video, 240 frames or images are extracted. Therefore, algorithms are implemented upon 240 images one by one automatically by the system. This serves as a major disadvantage because the system may need more computations [14].

Vehicle License plate identification is an essential stage in intelligent traffic system. In general, LPR comprises of four 
stages: Image acquisition and processing, License plate extraction, License plate segmentation and License plate recognition [13]. The video image processing technology is commonly used to identify vehicles by their license plates. Real time LPR play a major role in automatic monitoring of traffic rules and maintaining law enforcement on public roads. There are different techniques such as Sobel edge detector $[15,27]$, canny edge detection and morphological operators [16], skew correction [17] and color model [18]. One similarity between techniques discussed above is that they are implemented on single image and at day time only.

Canny edge detector $[19,25]$ is an edge detection operator that uses a multi-stage algorithm to detect wide range of edges in images. Mathematical morphology is used as tools to extract image components that are useful in representation and description of the object shape [16, 26]. Analysis is based on set theory, topology, lattice algebra, function etc. An existing system referred to as the Vehicle License Plate Localization and Recognition System was developed based on digital images and could be easily applied to commercial car park systems for the use of documenting access of parking services, secure usage of parking houses and also to prevent car theft. The license plate localization algorithm was based on a combination of morphological processes with a modified Hough Transform approach and the recognition of the license plates was achieved by the implementation of the feed-forward back propagation artificial neural network [20,24].

Vicar is a neural network based artificial vision system able to analyse the image of a car, locate the registration plate and recognize the registration number of the car. The main features of the system are: it controls the stability-plasticity behaviour, it enhances reliability of the threshold, both off-line and online learning, self-assessment of the output reliability and high reliability based on high level multiple feedbacks. It has an OCR engine [21]. Other methods demonstrate the use of dynamic neuro-fuzzy model which enhances the prediction capability of the system and hence gives accurate estimation for adoption and selection of the new technology. Combining the strengths of neural networks and fuzzy logic, the process was relatively simple, supports creation of high level pedagogical strategies and could be easily adapted to individual technological preferences. Compared to neural networks, the neuro-fuzzy methods provide models which can be interpreted by human beings. The system is in the form of familiar if-then rules implying easy selection with the operators [22].

The major setback of the existing systems are categorized into cost, efficiency, accuracy, ease to use, applicability, availability of resources etc. Advanced video detection is capable of monitoring several lanes simultaneously from a side mount position and delivers an image of the traffic situation, but performance suffers from poor lightening and bad weather conditions. In addition, real-time video detection is computationally expensive and relies on high-performance signal processing hardware, large amounts of memory, and high-bandwidth data links. The density of installation along a highway route is limited and may not reach the density necessary to acquire enough data for reliable traffic flow or intensity prediction

\section{TRAFFIC INTENSITY AND ESTIMATION}

Traffic estimation focuses on the population of vehicles speed, delays and queue lengths that result from the adoption of several traffic estimation strategies on individual roads, intersections and as well as toll gates. Intersection control and analysis tools throughout the world consider the population, speed, delays and queues as principal performance needed to be measured in order to determine the intersection level of service (LoS). This has to do with the evaluation of the adequacy of lane lengths, and the estimation of fuel consumption and emissions [23].

The rationale for concentrating on descriptive models is that a better understanding of the interaction between demand that is, arrival pattern and supply at traffic estimation is a prerequisite to the formulation of traffic control strategies. Estimation is based on assumptions regarding the characterization of the traffic arrival and service processes.

\section{A. Design Considerations}

The model is divided into three primary stages: data capture and pre-processing, data reduction and processing, and storage and feature analysis. In the data capturing stage, operations are performed to make data reduction and processing easier. The uncalibrated camera captures the incoming vehicle's data as video stream and converts it to indexed images. The first processing step is done by converting those images to grayscale. Data reduction stage involves eliminating unnecessary generated text and duplicate plate numbers as shown in figure 1. This stage includes finding the vehicle object in the frames, tracking it through the different frames and calculating the speed of the vehicles. All necessary information such as the arriving speed value, date, time, are also recorded in the database.

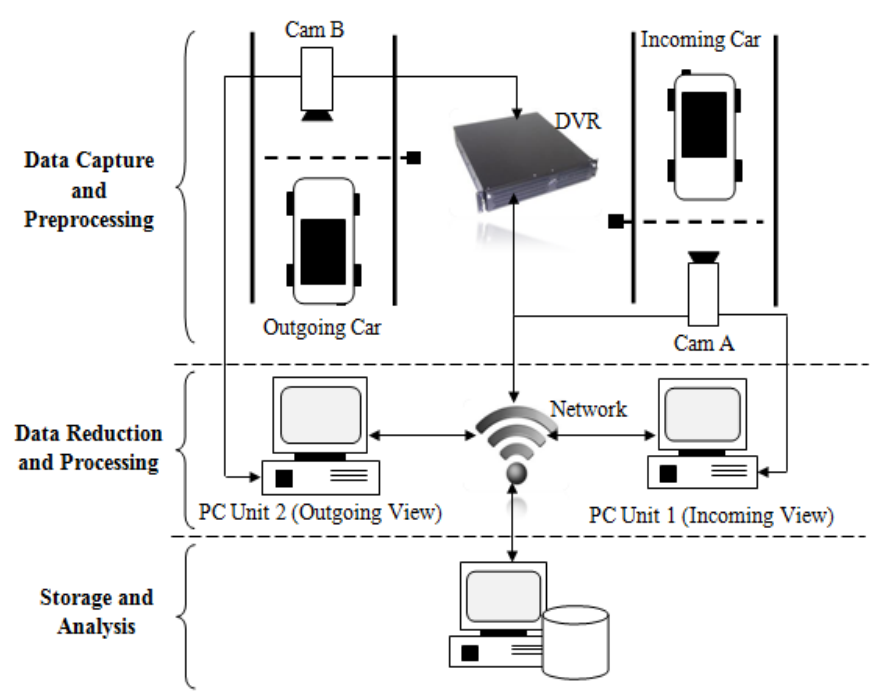

Fig. 1. A Strategic Traffic Monitoring System (STMS)

Capturing of video is done with the aid of the uncelebrated cameras. The cameras were strategically setup to capture the license plate as each car move across the camera lens. Closed Circuit TV (CCTV) was used for data capturing. The extraction of license is done with an Optical Character Reader (OCR) engine which is pre-installed on the system. The engine 
is responsible for capturing the available vehicle from a suitable frame of the video file.

\section{B. Traffic Monitoring System}

The Traffic Monitoring System is an encapsulation of basically two modules namely; Traffic information generation (TIG) module and the information analysis (IA) module. TIG is responsible for generating all the necessary information that will be needed to estimate the traffic during the analysis stage. It involve getting the video either in real time or pre-recorded from the Digital Video Recorder (DVR), detecting and extracting license plate number and storing up alongside the arrival speed, date and time in the database. Storage in the Traffic Monitoring System is categorized into two, storage of generated license plate number and other information about each vehicle after reading the video signal at real time. The instructions for TIG are given in algo 1 .

Algo 1: Algorithm for TIG

Step 1: Get video file from storage

Step 2: Convert video to still frames

Step3: $\quad$ Detect and Identify frame with valid license plate number

Step 4: Get speed value of the host vehicle

Step 5: $\quad$ Extract license plate number from frame

Step 6: Verify license plate number existence in database

Step 7: If (verification result $=$ true)

(Return "request check IN or OUT") Else

(Save Vehicle record (license_plate_number, speed value, date, time, cap_Time in database)

Step 8: $\quad$ saved license_plate_number with speed value, date and time and capture time.

Traffic Information Analysis (TIA) module makes reference to the database and gets all necessary information needed to generate traffic report about a selected data provided it exist in the database. To generate the report, information about the speed, total number of license plate, total time etc. are needed. Algo 2 describes the steps involved to illustrate the Traffic Information Analysis Module:

Algo 2: Algorithm for TIA

Step 1: Get date

Step 2: Verify the existence of record for the selected date

Step 3: If (Verification $=$ false $)$

(Return "No Record found") Else

(Proceed to next step)

Step 4: $\quad$ Total $\quad$ Speed $(\mathcal{E})=$ Total Speed + Speed Value $1+$ Speed Value $2+\ldots+$ speed $n$

Step 5: $\quad$ Average speed $=$ Total Speed $(\mathcal{E}) /$ Total number of existing License_plate_number (n)

Step 6: $\quad$ Total_time $(T)=$ Total time + cap_Time $1+$ cap_Time $2+\ldots+$ cap_Time
Step 7:

Traffic_Intensity Value $(\beta)=$ Total number of existing license plate $(n) /$ Total_time $(T)$

Step 8: Return Remark ("Minimal Traffic")

If $(n<=50$ per minute $)$

Else if ( $n>50$ per minute)

Return Remark ("Moderate Traffic")

Else if ( $n>100$ per minute)

Return Remark ("Intense Traffic")

Step 9: Generate Traffic report

Step 10: $\quad$ Associate generated report with bar chart.

The traffic monitoring system (TMS)flow structure shows the basic implementation of the algorithms in the license plate number and report generation presented in figure 2 and the use-case model for the TMS is described in figure 3. Figure 3 illustrates the overall accessibility of the different personnel and users of the system resources. It also illustrates the access control features of three major personnel namely: Administrator, Traffic Information Officer and the Security Officer. The administrator is considered to have no limits in accessing all the system resources. The administrator is responsible for the overall efficient and functional running of the system. He controls the activity of the system from both the front and back end. He gives report to the Traffic Information Officer (TIO).

Based on hierarchy, the Traffic Information Officer is considered the second level of access to the system resources. The access of the TIO is limited to viewing traffic report and information in the Database. The Security Officer has the lowest accessibility hierarchy to the security office in the front view.

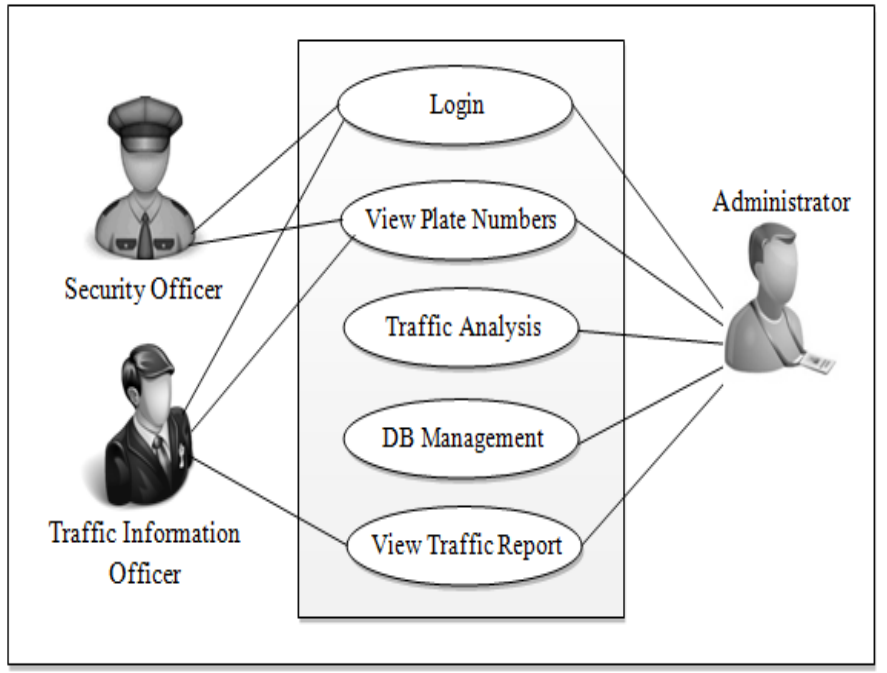

Fig. 2. License Plate number and Traffic information generation 


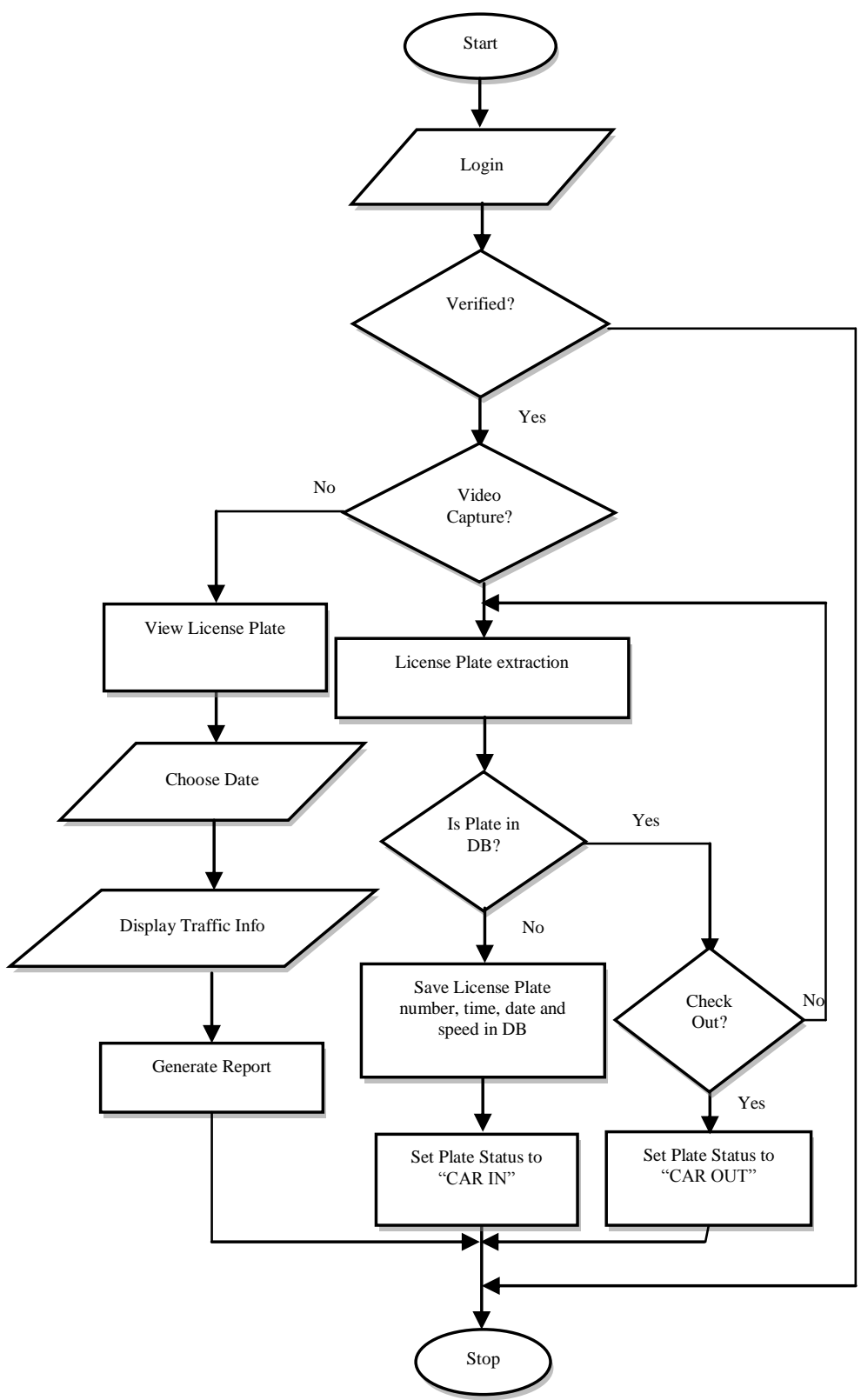

Fig. 3. Use case access control for Traffic Monitoring

\section{Toll Gates Traffic Intensity in Queueing Networks}

Traffic Intensity is a measure of the average occupancy of a server or resource during a specified period of time, normally a busy hour. It is measured in traffic units (erlangs) and defined as the ratio of the time during which a facility is cumulatively occupied to the time this facility is available for occupancy. If a toll gate is viewed as an interaction of services which vehicles pass through sequentially, then it is natural to model the system as a queueing network.

A queueing network can be represented as a directed graph shown in figure 4 in which the nodes denote the service points. Each point of the gate is represented by a separated service facility called a service centre. The ability of the service centre to provide services for any arriving vehicles does not depend only on the mean arrival rate but also on the pattern in which they arrive. The service time is the time a vehicle spends at the toll gate before proceeding. If the average duration of a service interaction between a service point and a vehicle is $\frac{1}{\mu}$, then $\mu$ is the service rate of the toll gate.

If there are more than one service points as observed in most toll gates the processing of vehicles can run parallel at the same time: one vehicle at each service point server. If there are fixed service points, say in this case 4 , then it is called multiple service points of servers $c=4$, each of which can service a vehicle at any time.

Figure 5 shows a multiple queue model in which vehicles at any point(s) which cannot receive service immediately, automatically waits in a buffer. The arrival rate $\lambda$ and the service rate are the most important features of a single queue. And the traffic intensity $\rho$ is denoted as

$$
\rho=\frac{\lambda}{c x \mu}
$$

A situation where the arrival points are different from the departures is considered. A transition diagram is described in figure 6 .

If the arrival rate at queue $i$ is $\lambda_{i}$ so also the departures will experience a Poisson departure stream with queue $i$ is $i+1$ and $\lambda_{i+1}$ also becomes $\lambda_{i}$ at another arrival. By decomposition principle, when the departure stream is split in this way, each of the resulting arrival streams will also be Poisson. If the service centres of any queue are analysed in this respect, expressing its input as a sum of output streams, what is obtained is called traffic equations. The traffic equation for figure 5 is as follows:

$$
\lambda_{1}=\lambda+q+\lambda_{2}
$$

$$
\lambda_{2}=p x \lambda_{1}
$$

One equation for each service point will give one unknown and this will end up with $n$ equations with $n$ unknowns. This traffic equation can be solved to get the arrival rate at each service point. This led to the utilization of the queue. The queue is being utilized whenever it is non-empty. Therefore, the Utilization $U$, is $\quad 1-\pi_{0}$ which implies $U=\rho$.

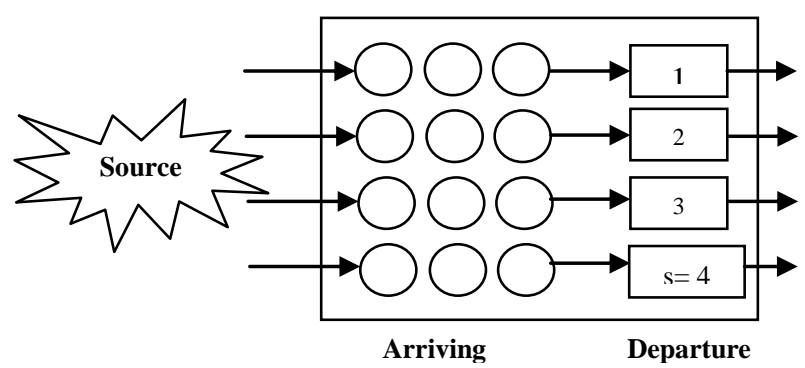

Fig. 4. An Toll gate Open Queueing Network 


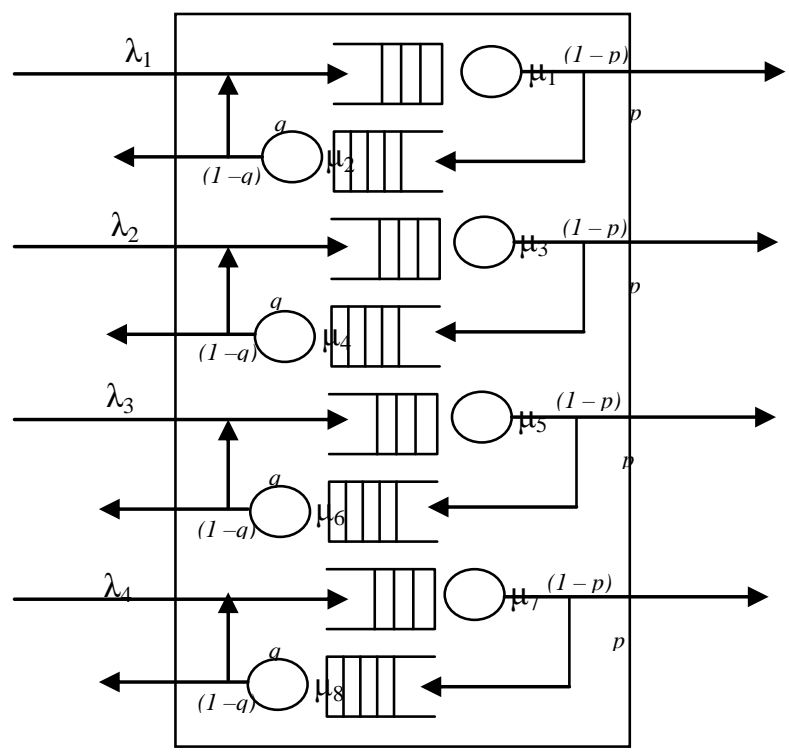

Fig. 5. Multiple Queues at Toll gate

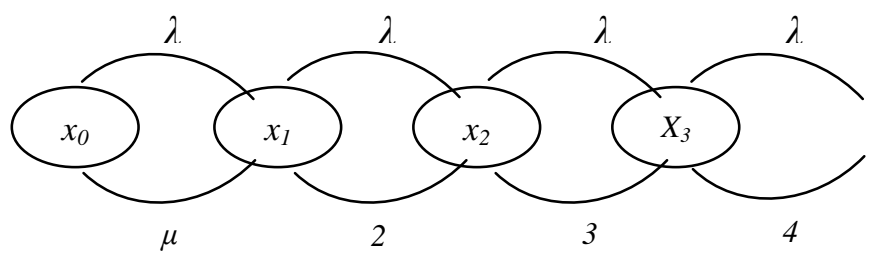

Fig. 6. An Toll Gate State Transaction Diagram for M/M/c

\section{IMPLEMENTATION AND RESULTS}

The integral subsystem of TMS consists of four main parts namely; Cameras, Computer (PC), Network Devices, Database. The cameras are uncalibrated video cameras that take in video signal as input data for the system. The camera reads in the video for processing in real-time and record overtime for batch processing. The PC hosts the software for processing and the network devices create connectivity between PCs indicating incoming and outgoing views. The unprocessed and processed information are stored by the database. The Object Oriented Paradigm (OOP) designed with the front end implemented with $\mathrm{C \#}$ with Microsoft .NET considering it flexibility, provides an interface for viewing the activities of vehicle at the entrance and it is capable of analysis after taking in all necessary data correctly. It also provides an easy approach for debugging and correction of errors. The backend is designed using the Microsoft SQL Server connected to the $\mathrm{C \#}$ modules.

The recorded is stored in the DVR and conversion of the video frame to an image was performed by the OCR engine. This process involves converting the single movie frame into an indexed image and an associated color map. A simple loop is used to repeat this operation for the entire movie, the dimension of the loop is decided by the length of the video and in turn return the total number of frames in the movie.
The Image Index is changed to its grays clay equivalent in order to prepare the index image for better identification of characters in the license place received. Bounding box extraction is followed by calling the property of the OCR engine to isolate the area of interest in the license plate image. The extraction of license is done with an OCR engine which is pre-installed on the system to be used. The engine is responsible for capturing the available license plate character from the suitable frame of the video file.

\section{A. Application Description}

The Administrator has complete control over the resources of the system. The access control is made possible with different accessing passkeys. The administrator's key enables all features of the application, but other keys only activates some features and disable others depending on who is logging in. Figure 7 consists of video feedback that shows camera recording in real time. The Information are: license per time, the capture time, the estimated arriving speed as well as the departure time of the vehicles. Figure 7 shows buttons used to access the list of captured license plate numbers. The buttons are enabled depending on who is logged in to access some control features. A multi-stage algorithm in canny edge detector was used to detect the vehicle images; result is shown in figure 8.

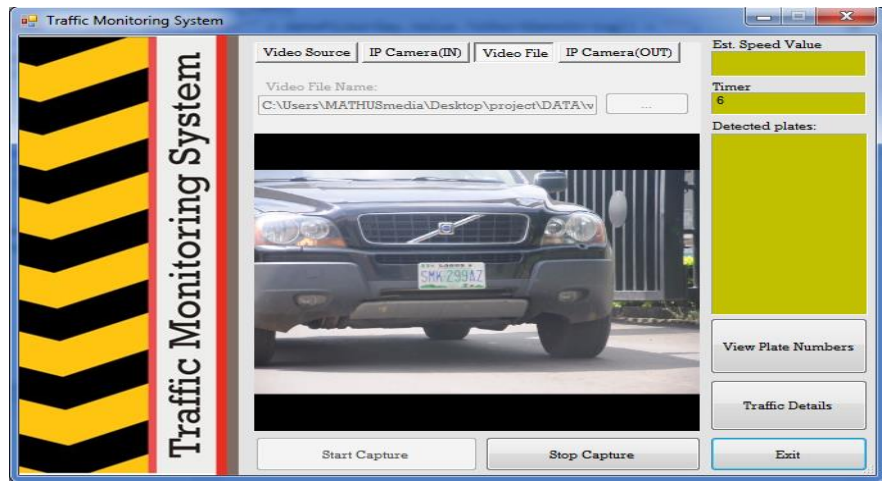

Fig. 7. Video Feedback of incoming Vehicles

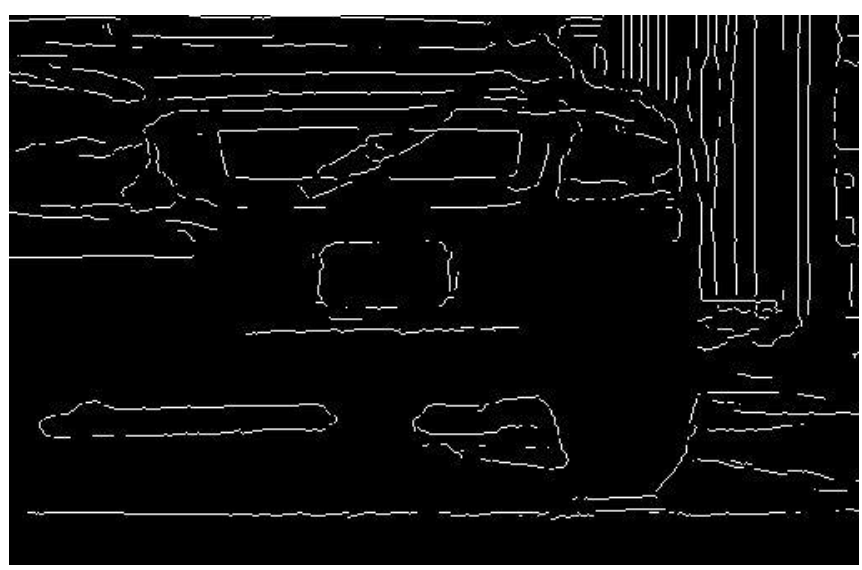

Fig. 8. Canny Edge Detection of the Vehicle on queue 


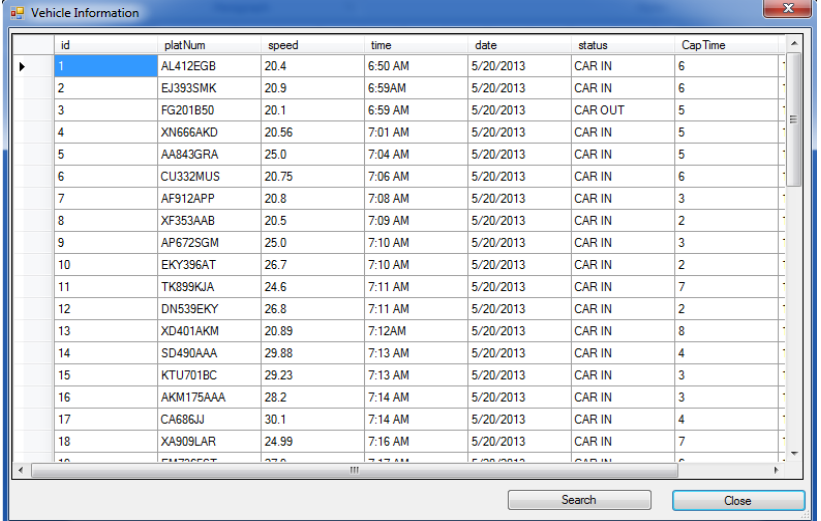

Fig. 9. Incoming Vehicles Information

The vehicle information is displayed in figure 9. It is accessed with view plate's button from the main interface. It provides information about the arriving speed, time, date and time of arriving and departing ones.

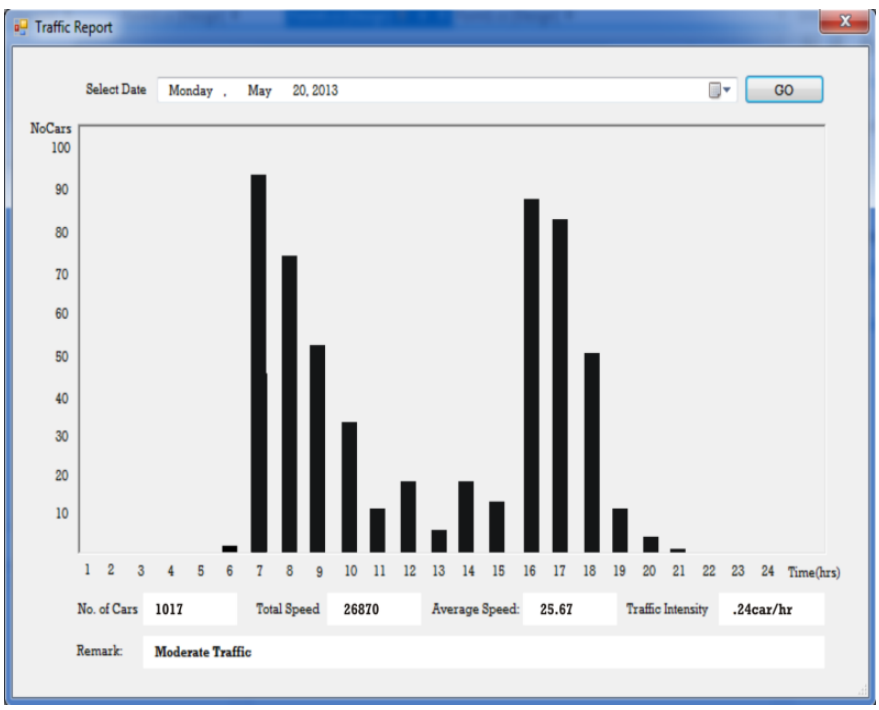

Fig. 10. Generated Report for Traffic Estimation

Figure 10 shows the traffic reports accessed with the traffic details button. The user selects the required date from the date selector box to generate the traffic report for the selected date. The histogram in figure 10 generated results for the number of cars per hour and the traffic information values for the total number of cars, total speed, average speed and the value for traffic intensity per day.

\section{B. Discussion}

The results were generated based on two days investigation carried out at the Federal University of Agriculture, Abeokuta, Nigeria (FUNAAB) ceremonial gate. The ceremonial gate officially open by 6:00am and closes by 10:00pm. The data were captured by pre-recording the traffic activity of the ceremonial gate with permission from the University Chief Security Officer (CSO). An uncalibrated mini DV video camera is used to capture the vehicle activities and giving an AVI format files, which are then batch processed by the software application. The result generated from the observation is given on tables 1 and 2 .
TABLE I. CAR PER HOURS FOR DAY 1

\begin{tabular}{ccc}
\hline Time Range & $\begin{array}{c}\text { No. of Incoming } \\
\text { Vehicles }\end{array}$ & $\begin{array}{c}\text { No. of } \\
\text { Outgoing } \\
\text { Vehicles }\end{array}$ \\
\hline 6:00am - 6:59am & 21 & 0 \\
7:00am - 7:59am & 142 & 6 \\
8:00am - 8:59am & 122 & 5 \\
9:00am - 9:59am & 81 & 10 \\
10:00am - 10:59am & 29 & 5 \\
11:00am - 11:59am & 24 & 10 \\
12:00pm - 12:59pm & 8 & 22 \\
1:00pm - 1:59pm & 12 & 25 \\
2:00pm - 2:59pm & 14 & 53 \\
3:00pm - 3:59pm & 12 & 60 \\
4:00pm - 4:59pm & 20 & 169 \\
5:00pm - 5:59pm & 9 & 83 \\
6:00pm - 6:59pm & 8 & 35 \\
7:00pm - 7:59pm & 3 & 20 \\
8:00pm - 8:59pm & - & - \\
9:00pm - 9:59pm & - & - \\
\hline
\end{tabular}

Figure 9 shows the traffic information at the FUNAAB ceremonial gate on 20th May 2013. It illustrates the trend at which the traffic varies with time. From the figure it could be observed that there is more vehicles coming between 7:00am and 9:00am and the number decreases as it move towards midday. At the outgoing end, it observed that there is gradual increase in the number of vehicles going out from midday towards evening. The same set up used on day 1 was repeated on day two.

Figure 10 illustrates the traffic information on day two. From the figure, it is observed that the trend was similar to the day one showing more cars arriving between 7:00am to 9:00am and most cars leaving between $3: 00 \mathrm{pm}$ to $6: 00 \mathrm{pm}$. But there exist a significant difference, it could be observed that on this particular day more cars came in early compared to day one which is around 6:00am to 7:00am.

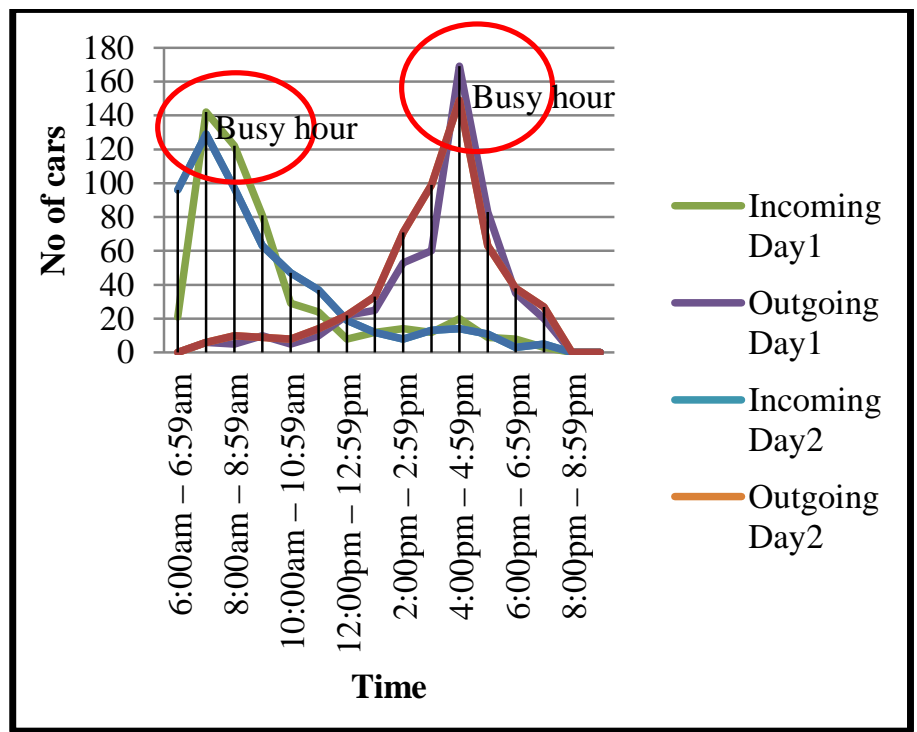

Fig. 11. Traffic Variations Showing the Busy Hours 
Figure 11 illustrates the overall activities at the ceremonial gate for the two days of observation. It could be observed that the peak of the graph is at 7:00 am - 9:00am on the incoming side and 3:00pm $-5: 00 \mathrm{pm}$ respectively. Hence it is assumed that the busy hours of the ceremonial gate are between 7:009:00am and 3:00-5:00pm respectively.

\section{Evaluation}

The evaluation is done using average percentage of success and the processing time. The throughput of the system was also examined to further examine the rate of achievement in determining level of success.

\section{1) Average Percentage of Success and Processing Time}

To examine the efficiency of the system, an average percentage of success tests with respect to average processing time is used. Average percentage of success is the percentage value of the total number of license plate generated by the application divided by the total number of vehicles counted manually ensuring $100 \%$ accuracy. To calculate the average percentage of success the formula is given as:

$$
\text { APS }(\%)=\frac{N L G}{N M C} \times 100
$$

APS $(\%)=$ Average Percentage of Success

$\mathrm{NLG}=$ Total number of license of license plate generated by application software

$\mathrm{NMC}=$ Total number of vehicle generated manually The result displaced in table 2 was derived using the above formula choosing sample time of 7:00am - 10:00am and 3:00pm - 6:00pm.

The results are shown in table 5 .

TABle II. Average Percentage of Success ANd Processing Time

\begin{tabular}{|c|c|c|c|c|c|c|}
\hline $\begin{array}{l}\text { Sample } \\
\text { Hrs. }\end{array}$ & $\begin{array}{c}\text { Incoming } \\
(\%)\end{array}$ & $\begin{array}{c}\text { DAY1 } \\
\text { Outgoing } \\
(\%)\end{array}$ & $\begin{array}{l}\text { Cap. } \\
\text { Time } \\
\text { (secs) }\end{array}$ & $\begin{array}{c}\text { Incoming } \\
(\%)\end{array}$ & $\begin{array}{c}\text { DAY2 } \\
\text { Outgoing } \\
(\%)\end{array}$ & $\begin{array}{l}\text { Cap. } \\
\text { Time } \\
\text { (secs) }\end{array}$ \\
\hline $\begin{array}{l}7: 00 \mathrm{am}- \\
7: 59 \mathrm{am}\end{array}$ & 999.9 & 100.0 & 4.1 & 99.2 & 100.0 & 4.3 \\
\hline $\begin{array}{l}\text { 8:00am - } \\
\text { 8:59am }\end{array}$ & 93.1 & 99.7 & 3.2 & 83.1 & 99.1 & 5.0 \\
\hline $\begin{array}{l}\text { 9:00am - } \\
\text { 9:59am }\end{array}$ & 86.1 & 100.0 & 5.0 & 100.0 & 99.0 & 4.3 \\
\hline $\begin{array}{c}\text { 3:00am - } \\
\text { 3:59am }\end{array}$ & 98.6 & 99.7 & 3.7 & 100.0 & 97.8 & 2.8 \\
\hline $\begin{array}{c}\text { 4:00am - } \\
4: 59 \mathrm{am}\end{array}$ & 100.0 & 89.3 & 2.1 & 99.5 & 99.3 & 3.1 \\
\hline $\begin{array}{l}5: 00 \mathrm{am}- \\
5: 59 \mathrm{am}\end{array}$ & 99.5 & 96.7 & 3.6 & 100.0 & 99.7 & 4.3 \\
\hline
\end{tabular}

\section{2) Throughput}

Figure 12 shows that the average percentage of success of the application capturing the saving license plate falls between $80 \%$ and $100 \%$. The failures are only due to factors such as faded license plate, low light etc. Figure 12 also shows that the maximum capture time of 5 seconds validate the speed and processing time of the application which is considered very fast under the required hardware specifications. The throughput for this process is drawn in figure 12. The result shows that at the busy hours; between 6am - 9am and 4pm$6 \mathrm{pm}$, the throughput is very high otherwise very low throughput is experienced.

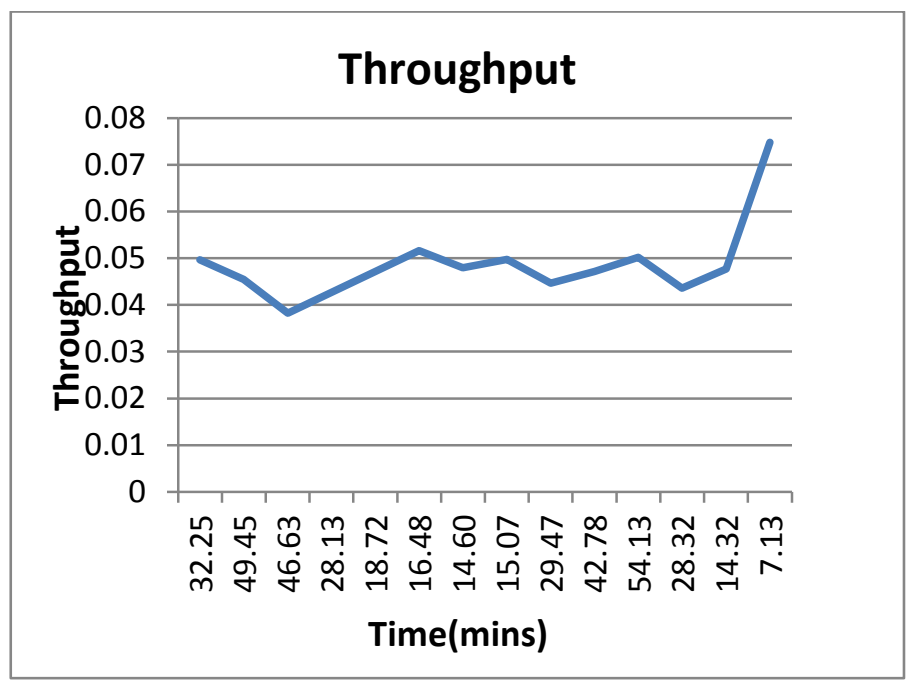

Fig. 12. Throughput versus Time

\section{CONCLUSION}

This research work presents a simple, low cost and efficient traffic monitoring system that allow the automated monitoring of traffic activity at a toll gate in which the system is implemented. The operation of TMS is based on the knowledge of the license plate number generated by the software application. The software makes reference to the number of generated license plate number with respect to time taken to generate all the number and in turn estimate the traffic intensity. This system is considered very efficient in detecting vehicle number plate from video files, either in real time or pre-recorded, all with the aid of the OCR engine in the application. It is highly effective in security areas like border crossing, entrance plaza, school gates, petrol station etc. It shows number plate automatically extracted from all images.

The estimated accuracy of capturing vehicle number plate from number of frames/images in a single video is $85 \%$ to $90 \%$; while getting desired result of number plate from overall video is $99 \%$. The advantage of this work is optimal in the provision of a simple automated, low cost, efficient and effective system of monitoring traffic and the idea of how license plate number can be used in the estimation of traffic intensity at toll gates. However, major problems posed are the inability of the application software to read the character in faded or complete washed off license plate.

Monitoring traffic is considered necessary in order to keep record and make traffic/vehicle related decisions. The limitations of the manual record keeping with the aid of the tally and the high cost of implementing the existing automated system gives room for the development of a simple and lowcost automated system to perform this tasks with more efficiency. TMS allows an automated license plate extractionto take place and in turn keeping other records about the vehicle hosting the license plate simultaneously. This reduces the manual labour and delays that often occur in the using of the manual tally system. This system of collecting entrances is eco-friendly and also results in increased entrance lane capacity. 
Moreover, the system can generate report automatically, which makes it is convenient for the user. The proposed system does not necessarily require a professional operator to work, it has a simple GUI. The Traffic Monitoring systemis efficiently used in the traffic surveillance for detection since it is capable of identifying individual vehicle with their license.

\section{REFERENCES}

[1] Khadijah, K. and Ismail, W., "Electronic Entrance Collection System Using Passive RFID Technology" Journal of Theoretical and Applied Information Technology, 2005, 70-76.

[2] Raadhikaa, L."The Universal Journal of applied Computer Science and Technology, UNIASCIT, 1, 1, 2011, 05-08.

[3] Friedman, J., “ Finding Mean Traffic Speed in Low Frame-RateVideo, IEEE Trans. Intelligent Transportation Systems 1, 2, 2005, 98-107.

[4] Lazaros G., George K., Elli P., “Automatic Estimation of Vehicle Speed from Uncalibrated Video Sequences", International symposium on modern technologies, education and professional practice ingeeodesy and related fields, 1-5, 2005

[5] Chetan, S. and Amandeep, K., "Indian vehicle license plate extraction and segmentation", InternationalJournal of Computer Science and Communication, Dept. of CSE, Patiala, 2, 2, 2011, 593-599.

[6] Lauren S. and Mariko B.,"Electronic Entrance Collection 2007.

[7] Xu, L., Wei, S. Ming-Lu, L. and Min-You, W., "Vehicle-based Sensor Networks for Traffic Monitoring", 2010.

[8] Xie, H., Kulik, L. and Tanin., E., "Privacy Aware Traffic Monitoring”, 2011, 1-7.

[9] Khali, P., Michael, C.W. and Shahriyar, H.,"Entrance Collection Technology and Best Practices", Project 0-5217:Vehicle/License PlateIdentification for Entrance Collection Application, 2007.

[10] Gabriel, N., Mitraszewska, I. and Tomasz, K, "The Polish Pilot Project of Automatic Entrance Collection System", Proceedings of the $6^{\text {th }}$ International Scientific Conference TRANSBALTICA, 2009.

[11] Ismail, M.S., KhairUl-Anwar, M.Y. and Zaida, A.Z., "Electronic Entrance Collection (ETC) Systems Development in Malaysia", PIAR CInternational Seminar on Intelligent Transport System (ITS) in Road Network Operations, 2006.

[12] Gunda, L., Lee M., Reginald G., Mhlanga, S. and Nyanga L., "Vehicle License Plate ", CIE42 Proceedings, Cape Town, South Africa 2012.

[13] Jameel, A.,"License Plate Recognition System, Thesis Presented to the Deanship of Graduate Studies", King Fahd University of Petroleum and
Minerals, 2003, 2-5.

[14] Kaur, H., Manviand Balwinder, S., "Vehicle License Plate Detection from Video Using Edge Detection and Morphological Operators". Singh International Journal of Engineering Research \& Technology ISSN: 2278-0181, 1, 9, 2012.

[15] Suri, P. K., Ekta, W. and Amit, V., "Vehicle Number Plate Detection using Sobel Edge Detection Technique", UCST, 1, 2, 2010, 178-182.

[16] Vasudevan, Shriram, K., Dharmendra, T. and Sivaraman, R., "Automotive Image Processing Technique using Canny's Edge Detector" International Journal of Environmental Science and Technology, 2010, 2632-2644.

[17] Manchikalapudi, V., "Skew Correction and Localisation of Number Plate Using Hough Transform" UCST, 2011, 472-476.

[18] Deb, K. and Hyun Jo, K. "Segmenting the License Plate Region using Color Model", University of UlsanSouth Korea, 2009, 401-418.

[19] Little, J. D. C.. "A Proof for the Queuing Formula: $L=\lambda W "$ " Operations Research9, 3,1961, 383-387.

[20] Ganapathy, V. and Lui, D. A., "Malaysian Vehicle License Plate Localization and Recognition System", School of Engineering, Monash University of Malaysia, 2007.

[21] Draghici, S., “ A Neural Network-Based Artificial Vision System for License Plate Recognition",International Journal of Neural System. 8, 1, 1997, 113-126.

[22] Kalbande, D.R., Signal, P., Denotable, N., Shah, S. and Tampa, G.T.,"An Advanced Technology Selection Model using Neuro Fuzzy Algorithm for Electronic Entrance Collection System", International Journal of Advanced Computer Science and Applications, 2, 4, 2011.

[23] Rouphail N., Tarko, A. and Li, J., "Traffic Flow at Signalized Intersections" Transportation Research Record, 2009, 9-32.

[24] Dailey, D.J. And Li, L “An Algorithm to Estimate Vehicle Speed using Uncalibrated Camera", IEEE, vol 4,8, 3-12. 1999.

[25] Leung, T. and Malik, J., "Representing and recognizing thevisual appearance of materials using three-dimensional textons. IJCV, 43, 1, 2001, 29-44.

[26] Lowe, D., " Distinctive image features from scale-invariant keypoints". IJCV 2004.

[27] Lowe, D. G., “ Object recognition from local scale-invariant features. InICCV, 1999,1150-1157.

[28] Paulev_e, L., J_egou, H., and Amsaleg, L., " Locality sensitive hashing:a comparison of hash function types and querying mechanisms. Pattern Recognition Letters,31,11, 2010, 1348-1358. 